\title{
LECTURAS DEL PERONISMO Y LA CULTURA EN CONTORNO $(1953-1959)^{1}$
}

\author{
«Bajo la égida del régimen peronista, todas las relaciones entre \\ los grupos sociales se vieron súbitamente redefinidas, \\ y para advertirlo bastaba caminar las calles o subirse a un tranvía». \\ Tulio Halperin Donghi, \\ La larga agonía de la Argentina peronista (1992)
}

Palabras clave: revista Contorno, cultura, peronismo, Masotta, Halperin Donghi

\section{Introducción}

Entre los años de 1953 y 1959 se edita, en Buenos Aires, la revista Contorno, dirigida, principalmente, por los hermanos Ismael y David Viñas ${ }^{2}$. La publicación constará de

1 Este artículo es una reescritura de la primera parte del trabajo presentado para el seminario de posgrado Operaciones críticas en el campo literario argentino (1955-2000), dictado por la Dra. María Coira en el marco de los doctorados en Letras y en Historia de la Universidad Nacional de Mar del Plata, Otoño de 2011: «Política y cultura en Contorno: el peronismo y el frondizismo en cuestión». Agradezco a la profesora las conversaciones sostenidas cada jueves sobre estos temas y problemas.

2 Ofrecemos aquí el índice detallado de la revista:

№ 1 (nov. 1953): Sebreli, Juan José, Los martinfierristas: su tiempo y el nuestro; Viñas, Ismael, La traición de los hombres honestos; Viñas, David, Milonga; Prieto, Adolfo, A propósito de Los Ídolos; Sanromán, V. (seud. de Ismael Viñas), «Ladrones de bicicletas» o la decepción frente al cine; Angeli, Héctor Miguel, El despiadado.

№ 2 (may. 1954): Dedicado a Roberto Arlt. Conte Reyes, Gabriel (seud. de David Viñas), La mentira de Arlt; Viñas, Ismael, Una expresión, un signo; Elorde, Ramón (seud. de David Viñas), Erdosain y el plano oblicuo; Solero, F. J., Roberto Arlt y el pecado de todos; Gorini, Juan José (seud. de David Viñas), Arlt y los comunistas; Molinari, Marta C. (seud. de David e Ismael Viñas), Roberto Arlt: una autobiografía; Kiernan, Fernando, Roberto Arlt, periodista; Sánchez Cortés, Diego (seud. de David Viñas), Arlt - Un escolio; Arrow, Jorge (seud. de Ismael Viñas), Arlt - Buenos Aires; Gigli, Adelaida, El único rostro de Jano; De las obras y los hombres. Correas, Carlos, H. A. Murena y la vida pecaminosa; Prior, Aldo, Rodolfo Kusch y la seducción de la barbarie; Goutman, Ana A., Estela Canto: ¿una novela?.

№3 (sep. 1954): Gigli, Adelaida, Victoria Ocampo: V. O.; Sebreli, Juan José, Manuel Gálvez y el sainete histórico; Kusch, Rodolfo, Inteligencia y barbarie; Viñas, David, Otros tres novelistas argentinos por orden cronológico; Viñas, Ismael, Engañado adanita; Solero, F. J., Responso; Gibaja, Regina, La mujer: un mito porteño; Correas, Carlos, El revólver.

№ 4 (dic. 1954): Dedicado a Martínez Estrada. Weinbaum, Raquel (seud. de David Viñas), Los ojos de Martínez Estrada; Viñas, Ismael, Reflexión sobre Martínez Estrada; Suevo, Orlando, Bibliografía de Martínez Estrada; Kusch, Rodolfo, Lo superficial y lo profundo en Martínez Estrada; Solero, F. J., Primera aproximación a Martínez Estrada; Viñas, David, La historia excluida: ubicación de Martínez Estrada; Gigli, Adelaida, La poesía de Martínez Estrada: Oro y Piedra para siempre.

№ 5/6 (sep. 1955): Dedicado a la novela argentina. Contorno, Terrorismo y complicidad; Weinbaum, Raquel (seud. de David Viñas), Los dos ojos del romanticismo; Solero, F. J., Eugenio Cambaceres: primer novelista argentino; Pagés Larraya, Antonio, Julián Martel y la ciudad hostil; Aseef, Víctor, Esquema de Sicardi; Steffen, Guillermo, Bosquejo de nuestra propia expresión: Payró; Viñas, Ismael/Noé Jitrik, Enrique Larreta o el linaje; Molinari, Marta C. (seud. de David e Ismael Viñas), Manuel Gálvez: el realismo impenitente; Viñas, David, Be- 
diez números y dos Cuadernos (o números especiales) que describen un cambio de tono desde su primera parte -los cinco primeros números, básicamente destinados a la crítica cultural y literaria- hasta su zona final - en la que el interés por la política local se vuelve un imperativo.

El período histórico que va desde la última etapa de la segunda presidencia de Juan Perón, pasa por el gobierno militar que derrocó a este presidente (Revolución Libertadora) y que culmina con las elecciones posteriores y el consecuente triunfo de Arturo Frondizi en un contexto de proscripción para el peronismo, revela muchos matices a la hora de observar las relaciones del poder político con los escritores e intelectuales, por una parte, y con las instituciones tradicionalmente a cargo de los espacios culturales, esto es, la universidad, las editoriales, las academias, por otra. En este sentido, es claro que Contorno provoca una ruptura en el ámbito de las letras por varias cuestiones relacionadas, en primer lugar, con el universo interno de la crítica literaria y, en segundo término, por la misma adhesión a un espacio político, el frondizismo, que venía a ser equidistante entre el antiperonismo conservador -cuyo epítome es la revista dirigida por Victoria Ocampo, Sur-y los escasos intelectuales que explícitamente habían adherido sin más al proyecto peronista (Leopoldo Marechal, entre otros).

Por otra parte, es insoslayable la observación de que las corrientes de izquierda en la Argentina, partícipes de fervorosos debates respecto de los intereses nacionales, latían, de alguna manera, al calor de los episodios internacionales y en lo que a las distintas corrientes del marxismo o simpatizantes, en el marco de las lecturas -la althusseriana, la sartreana o, más tarde, la gramsciana- respecta. Así, el lenguaje de los intelectuales,

nito Lynch: la realización del Facundo; Viñas, Ismael, Güiraldes; Conte Reyes, Gabriel (seud. de David Viñas), Realismo, virtuosismo y técnica: Juan Goyanarte; Gibaja, Regina, Ernesto L. Castro y la novela social; Arrow, Jorge (seud. de Ismael Viñas), Enrique Wernicke: la poesía de las chacras; Rozitchner, León, Comunicación y servidumbre: Mallea; Sánchez Cortés, Diego (seud. de David Viñas), Verbitsky, Onetti: el hombre urbano, el hombre universal; Pandolfi, Rodolfo M., Mujica Láinez y el gran cambio; Jitrik, Noé, Adán Buenosayres: la novela de Leopoldo Marechal; Sanromán, V. (seud. de Ismael Viñas), Pablo Rojas Paz, viejo martinfierrista; Gigli, Adelaida, Algunos libros, algunas mujeres; Jitrik, Noé, Los comunistas (Manauta, Barletta, Yunque, Varela); Curi, Jorge, Un ortodoxo: Carlos Ruiz Daudet; Gargano, Julio, Los nuevos; Discusión. Troiani, Osiris, Fin de un diálogo de sordos; Alcalde, Ramón, Imperialismo, cultura y literatura nacional.

No 7/8 (jul. 1956): Contorno, Peronismo, ¿y lo otro?; Rozitchner, León, Experiencia proletaria y experiencia burguesa; Troiani, Osiris, Examen de conciencia; Viñas, Ismael, Miedos, complejos y malos entendidos; Halperin Donghi, Tulio, Del fascismo al peronismo; Pandolfi, Rodolfo M., 17 de octubre, trampa y salida; Prieto, Adolfo, Peronismo y neutralidad; Viñas, David, ¡Paso a los héroes!; Masotta, Oscar, Sur o el antiperonismo colonialista; Sebreli, Juan José, Aventura y revolución peronista. Testimonio. De las obras y los hombres. Sanromán, V. (seud. de Ismael Viñas), La fiesta del monstruo; Verón Thirión, Ernesto, Víctor Massuh o el encubrimiento de América; Jitrik, Noé, Guibert: un poeta con geografía; Molinari, Marta C. (seud. de David Viñas), Rosaura a las diez, Premio Kraft; Alcalde, Ramón, Catecismo político para un nuevo Uriburu. Ayer, hoy y mañana, de Mario Amadeo. No 9/10 (jul. 1956): Contorno, Análisis del frondizismo; Rozitchner, León, Un paso adelante, dos atrás; Viñas, Ismael, Orden y progreso; Halperin Donghi, Tulio, El espejo de la historia.

Contorno. Cuadernos $\mathrm{N}^{\circ} 1$ (jul. 1957): Lucha de clases, laicismo: examen para la izquierda. Viñas, Ismael, Un prólogo sobre el país; Alcalde, Ramón, La Iglesia argentina: instrucciones para su uso; Rozitchner, León, Lucha de clases, verificación del laicismo; Contorno, Resollando por la herida ...; Sin firma, Para colaborar con Contorno. Sin firma, Defensa y opresión de la democracia.

Contorno. Cuadernos № 2 (feb. 1958): El fracaso del Panamericanismo. Viñas, Ismael (por Contorno), Una opción. Subordinación, independencia, desarrollo; Muñoz de Liceaga, Marisa, El fracaso del panamericanismo. La Conferencia Económica Interamericana y las inversiones estadounidenses. 
la política y la cultura se va entremezclando y repensando en forma permanente. Este clima será propicio para el carácter polémico de ciertos debates y, desde luego, para los sucesivos «ajustes de cuentas», para decirlo con Carlos Correas, que los propios protagonistas realicen con sus jóvenes iniciativas, los derroteros personales, distanciamientos intelectuales y afectivos; en fin, con los balances que, en décadas posteriores, suscitó este momento crucial de la reflexión intelectual argentina.

\section{Contorno: una cita con nuevas formas de decir}

Desde el primer número de Contorno, aparecido en noviembre de 1953 se puede advertir esta voluntad constante de concebir al intelectual como un elemento denuncialista y polémico. Y, por supuesto, un intelectual comprometido con su tiempo ${ }^{3}$. El primer gesto de Contorno es la reconocida reivindicación de Ezequiel Martínez Estrada, un escritor aún vivo en ese momento y que después de haber sido fundamental, por sus escritos sobre el «ser nacional», en el centro del paisaje cultural argentino, se iba alejando hacia los márgenes. ¿Qué les atrajo a los contornistas de Martínez Estrada? En primer lugar, ese tono denuncialista que caracterizaba sus ensayos más célebres -Radiografía de la pampa, Muerte y transfiguración del Martín Fierro. Por otra parte, el gesto de elegirlo es de por sí provocativo: los contornistas estaban diciendo «no somos Ricardo Rojas», quien sostenía que a la historia de la literatura ingresaba sólo lo que estuviera canonizado por la muerte. Esta elección constituyó una forma de inclinar la balanza hacia otras miradas de la historia y la literatura, en las que la escritura de lo contemporáneo y la revisión y reescritura del pasado se volvieron fundamentales.

Al número dedicado a Martínez Estrada lo había precedido uno destinado a exaltar la figura de Roberto Arlt. Tan en el centro de la literatura y la crítica argentinas actuales, en el momento de la irrupción de Contorno Arlt era poco menos que un marginal de la escritura, un escritor malo, cuya escritura expresaba sólo desequilibrios, ignorancia y resentimientos. Los estudios sobre su obra se sucedieron sin descanso hasta el día de hoy, y las distancias y diferencias que ponen en paralelo para denostar a Arlt o a Borges según quién sea el observador ya son interminables. Es que sin que se efectuara una oposición o un enfrentamiento real, entre esos escritores contemporáneos y anacrónicos al mismo tiempo, podemos decir que se trató de una operación crítica exitosa por parte del grupo contornista, más allá de que alguno de sus miembros -tal el caso del grupo marginal (pero de gran impacto) a Contorno, Juan José Sebreli, Carlos Correas y Oscar Masotta- se dedicaran a estudiar a Arlt, descubrieran en él cierta caracterización de sí mismos y no despreciaran por ello, sino muy por el contrario exaltaran, la escritura de Borges ${ }^{4}$.

En términos generales, sin embargo, la cuestión de oponer Borges a Arlt tiene su primera raíz en el rechazo abierto y explícito de los contornistas por el grupo Sur y los

Ismael Viñas (director), Adelaida Gigli, Oscar Masotta, David Viñas (y sus múltiples heterónimos), León Rozitchner, Adolfo Prieto, Juan José Sebreli, Ramón Alcalde y Regina Gibaja asumirán como propia la figura del intelectual comprometido llamado a «desgarrarse de su clase» y asumir las voces de aquellos que jamás hojearán la revista (Croce, 1996: 75).

4 No es el caso de David Viñas quien repitió, toda su vida, «Borges nunca me interesó», con gran desparpajo al decir de Beatriz Sarlo. 
martinfierristas (el grupo de los vanguardistas que publicaba en la revista Martín Fierro). Incluso en este terreno es dable señalar que tampoco era del todo así en cuanto a los intercambios con la revista de Ocampo y porque el universo internacionalista de opciones que proponía Victoria era, sin duda, en algún punto compartido - la introducción del sartrismo en la Argentina se debió, principalmente, al grupo Sur ${ }^{5}$. Estas opciones o determinaciones dentro del campo literario -restitución de Martínez Estrada, primero, de Arlt después, el abierto rechazo a la crítica canónica y canonizadora de Sur- tienen su expansión, por así llamarla, en otros planos, como la expresión de la crítica desde un espacio de fuerte legitimación como lo era la academia. Los contornistas rechazan el supuesto saber académico, más allá, -nuevamente más allá- de que muchos de ellos provenían de la universidad, actuaron en ella y formaron parte de su vida en muchas ocasiones, en especial, durante la dictadura de Aramburu, el frondizismo y, en años posteriores a todas esas dictaduras, en la universidad de la democracia ${ }^{6}$.

\section{La lectura del peronismo}

La clave que nos interesa señalar es la disyuntiva en la que este grupo se siente frente al fenómeno del peronismo y a la avanzada de la Revolución Libertadora, la contemplación negativa de la izquierda frente a esto y el brindis de Sur frente a la caída de Perón. Observaremos en este punto tres miradas puntuales: la de la editorial de Contorno, la reflexión de Oscar Masotta y, por último, el análisis de Tulio Halperin Donghi. Estos dos articulistas de la revista construyeron carreras intelectuales disímiles -el primero en el ámbito de la crítica literaria y del psicoanálisis; el segundo es, hasta el día de hoy, considerado uno de los mayores historiadores de Argentina- pero de fundamental impacto en sus respectivas disciplinas. Estos artículos de «juventud» difieren de los procesos de madurez, aunque, como se verá, en algunas cuestiones se vislumbran aquí las raíces de los ulteriores desarrollos disciplinares.

\subsection{La opinión de la revista a través de su editorial}

«Las revistas culturales son, pues, un modo de organización de la intelligentsia y engendran microclimas propios. A través de ellas pueden seguirse las batallas de los intelectuales [...] y hacer el mapa de sensibilidad intelectual en un momento dado.»

Carlos Altamirano, Intelectuales. Notas de Investigación (2006)

Muchos años después de la creación de Contorno y poco antes de morir, David Viñas, un intelectual reconocido y partícipe medianamente los medios de comunicación, acuñó la frase «Un intelectual no puede ser oficialista». En los años iniciales de Contorno, esta idea es rectora. La revista, salida durante el peronismo, habla de literatura, no de

\footnotetext{
Saint Genet. Comédien et martyr, Paris, Gallimard, de 1952, por ejemplo, había llegado a ser leído en la Argentina merced a las gestiones y traducciones del grupo Sur.

6 Ismael Viñas fue secretario de José Luis Romero, interventor de la UBA, y Ramón Alcalde actuó en el Ministerio de Educación en la Provincia de Santa Fe durante el período frondizista, por ejemplo.
} 
Perón: los participantes son todos antiperonistas, incluso de una fuerte militancia, aunque la prudencia los llevaría a concentrarse en la literatura. No es funcional a las políticas gubernamentales. Pero, en su número de julio de 1956, es decir, posteriormente a la revolución de septiembre, el tema central es el peronismo; en los tiempos de la proscripción y la dictadura de Aramburu, que había prohibido inclusive la mención del nombre de Perón. Hablan de peronismo en el momento en el que Rodolfo Walsh publica Operación Masacre, es decir, dando por evidente la captación crítica de que el tono del momento pasaba por reflexionar acerca de ese fenómeno de masas cuyos derroteros llegarán hasta la política y la cultura actual y es, desde todo punto de vista, el hecho histórico y social más traumático del siglo XX argentino.

En el artículo editorial, son interesantes las correspondencias que aparecen a partir de la funcionalidad del epígrafe inicial del número. Se trata de una cita, escrita en 1839 y 1840, extraída de los escritos póstumos de Juan Bautista Alberdi, en la que el jurista argentino denunciaba la lucha facciosa entre antiguos unitarios y federales, entre colorados y celestes y los «inmensos males» que esta reyerta había provocado. En medio de los desaciertos que señalaba Alberdi, no era el menor, por cierto, la distancia que la lucha intestina llevó a sus partícipes a desconocer su medio de acción, a las masas y de allí su decrépito anacronismo. Dice Alberdi:

Luego nos argüirán, para condenar todo lo que contiene nuestro país de glorioso y distinguido en personajes políticos y literarios, ¿por qué habéis recorrido los dos partidos que le constituyen con el acto de reprobación en la mano y tirado indistintamente sobre ellos? ¿Qué es, pues, lo que reís? ¿A qué partido pertenecéis vos? ¿En provecho de qué idea, de qué sistema, de qué gentes escribís? ... Yo contestaré: hace muchos años que persigo a las dos fracciones en que se ha dividido la generación pasada de mi país, porque no nos han hecho sino inmensos males: la colorada por sus crímenes; la celeste por su inepcia ... Juzgo al pasado con severidad y llamo al porvenir a sucederlo. Digo que es tiempo de que el país cuide de no confiar la menor de sus tentativas de regeneración a hombres que no harán sino malograrlas, porque han perdido la fe y la disposición al sacrificio, y han cesado, sobre todo, de comprender los instintos y los medios de acción de nuestras masas: han pasado como su tiempo. (Alberdi, Acontecimientos del Plata. Póstumos, XV, 1900)

Esta lectura es concomitante con la de otros integrantes de la generación romántica. Recordemos, por ejemplo, a Echeverría en la alegoría que propone de «El matadero de la Convalecencia». Las reticencias del toro y su enfrentamiento con los carniceros federales provocan la muerte de un niño observador, personaje que simboliza el futuro patrio:

-Allá va -gritó una voz ronca, interrumpiendo aquellos desahogos de la cobardía feroz-. ¡Allá va el toro!

- ¡Alerta! ¡Guarda los de la puerta! ¡Allá va furioso como un demonio!

Y en efecto, el animal acosado por los gritos y sobre todo por dos picanas agudas que le espoleaban la cola, sintiendo flojo el lazo, arremetió bufando a la puerta, lanzando a entre ambos lados una rojiza y fosfórica mirada. Dióle el tirón el enlazador sentando su caballo, desprendió el lazo del asta, crujió por el aire un áspero zumbido y al mismo tiempo se vio rodar desde 
lo alto de una horqueta del corral, como si un golpe de hacha la hubiese dividido a cercén, una cabeza de niño cuyo tronco permaneció inmóvil sobre su caballo de palo, lanzando por cada arteria un largo chorro de sangre.

-Se cortó el lazo - gritaron unos-: jallá va el toro!

Pero otros deslumbrados y atónitos guardaron silencio porque todo fue como un relámpago.

Desparramóse un tanto el grupo de la puerta. Una parte se agolpó sobre la cabeza y el cadáver palpitante del muchacho degollado por el lazo, manifestando horror en su atónito semblante, y la otra parte compuesta de jinetes que no vieron la catástrofe se escurrió en distintas direcciones en pos del toro, vociferando y gritando ... (Echeverría, 1991: 133)

Entonces, la reflexión de Alberdi como intelectual joven no se halla aislada sino que se corresponde con su grupo generacional. Él y su grupo a su vez guardan una relación crítica y equidistante respecto de los dos partidos tradicionales de su tiempo: son, a fin de cuentas, hombres de letras. Y, por otra parte, esos partidos han provocado la disolución de proyectos alternativos que procuraran un auténtico avance en la construcción social. Por último, -la cita en particular, pero la entera obra de Alberdi nos lo dice-, su preocupación como hombre de pensamiento fue, una y otra vez, desbrozar lo verdadero en los sucesos y así procurar una visión que cooptara lo real, en ese sentido expresado, una distancia crítica.

Esta cita, entonces, guarda todas las correspondencias necesarias para situar la mirada concreta del editorial de Contorno, presumiblemente de David o de los hermanos Viñas y no será la única versión posible de aproximación al peronismo en el número en cuestión. Una vez más, como se había expresado ya desde el primer número (Sebreli), los contornistas se identifican con la generación romántica de 1837 y casi cien años después reeditan parte de su legado ideológico - de allí, por ejemplo, las antipatías hispanistas de Contorno frente a la crítica tradicional de principios de siglo cuyo carácter hispanizante escondía el marcado acento xenófobo por el proceso inmigratorio. En este sentido, reproducen en primera instancia el rechazo por el binomio peronismo/anti-peronismo y se distancian de cada una de estas posturas. Dirán: «no solamente no habernos entregado al peronismo, sino tampoco al antiperonismo» ${ }^{7}$. Piensan, como Alberdi en su tiempo, que no se debe inclinar, en tanto intelectual al oficialismo de turno ...

Sin embargo, agregan algo a esta mirada correlativa con la generación romántica y es el carácter autocrítico que sostiene todo el artículo respecto de cómo este grupo vivió el peronismo ${ }^{8}$. Esto los lleva a analizar la matriz por cierto ambigua del proceso y sopesar los pros y los contras en cada caso.

Ha dicho Beatriz Sarlo: «Como los románticos en 1837, Contorno se propone ser la síntesis de los dos partidos, que son, también, dos miradas. La cuestión está en cómo dirigirlas (y desde dónde) para que, en lugar de una percepción estrábica cuya condena es reproducir su doble objeto [...], las perspectivas sean precisamente eso: líneas imaginarias de organización de lo real, líneas de lectura y de escritura.» (Sarlo, 1983)

8 Como lo señala Viñas, con uno de sus heterónimos, «En nuestro país se han enfrentado la barbarie y la decadencia. Los argentinos que aún no tienen conciencia - conciencia de sí mismos- por y para una clase, por y para una nación- y los que ya la han perdido. Las mayorías crédulas y las minorías cínicas. ¿Cuál era nuestro bando? Ninguno de los dos. ¿Pero dónde hallaríamos aliados? En los dos sin duda.» (O. Troiani 1956). 


\subsection{Un peronista idealista: el caso Masotta}

Así denominó Carlos Correas a Oscar Masotta, ambos integrantes del grupo Contorno. Un peronista, Masotta, que ha cuestionado la mirada del peronismo elaborada por la alta burguesía argentina que el grupo Sur representaba y a la que interpela en el artículo «Sur o el antiperonismo colonialista» publicado en Contorno en el número 7-8 de julio de 1956, lo que constituye su segunda y última aparición en la revista. Este artículo en particular y el número en su conjunto oficia, en rigor, como una respuesta al número 237 de Sur, de noviembre de 1955, donde Victoria Ocampo llamaba a la unidad antiperonista para reconstruir la situación en que el peronismo había dejado, según ella, a la nación:

Lo que acabamos de vivir ha demostrado la magnitud del peligro. [...] aprovechemos una lección tan cruel y que hubiera podido serlo aún más si el impulso de algunos hombres que se jugaron la vida no hubiera intervenido de manera milagrosa. [...] ayudémoslos con toda nuestra buena voluntad, con toda nuestra preocupación de verdad y de probidad intelectual. (V. Ocampo, 1955)

En esta contestación, Masotta relaciona la burguesía antiperonista con el pensamiento colonialista y dominante de la revista de V. Ocampo. De un modo casi estructualista, analiza la extensión de los términos acuñados por Sur para defender sus bastiones culturales -espíritu, verdad- y cómo se ven parcializados a partir de las denuncias de, por ejemplo, los campos soviéticos y el aval explícito e implícito de las modalidades de colonialismo norteamericano y desenmascara los términos fijados como «golpe democrático» -el que derrocó a Perón, según Sur- en clave netamente anti-obrera ${ }^{9}$. Como ha señalado Marcela Croce, «el No 7/8 de Contorno es la contrapartida exacta del N 237 de Sur» (Croce, 1996) porque la revista de los hermanos Viñas conjugará esa mirada sartreana que compromete al intelectual a una posición activa frente a los problemas sociales con su propio posicionamiento intelectual, opuesto al «antiperonismo colonialista» postulado por Masotta.

\subsection{Halperín Donghi y su primeras lectura del peronismo}

«Desde el Fascismo al Peronismo», el artículo escrito por Tulio Halperín Donghi, nos ofrece una lectura que tiene todo el formato de querer contestar los términos en que el gobierno recientemente derrocado era valorado por la enorme mayoría del arco antiperonista y que se había convertido, a la sazón, en la visión oficial de la Revolución Libertadora: el peronismo como versión vernácula del fascismo. Pero no. La tarea de Halperín, en su artículo de Julio de 1956, no cumple otro objetivo que corregir y perfeccionar esa lectura. Porque si considerar al peronismo como fascismo tiene una dosis degradante, más aún teniendo en cuenta lo reciente de la finalización de la guerra mundial

\footnotetext{
Masotta también interviene en una publicación ya de mayor envergadura política, como lo es Clase obrera, dirigida por Rodolfo Puiggrós. Había publicado en 1955 «La tragedia del hombre en el radicalismo» y en octubre de 1955 publica «El proletariado en la alternativa». Clase obrera se subtitulaba órgano del movimiento obrero comunista y era, en un sentido laxo y complejo «afín» al movimiento peronista.
} 
y las características aberrantes que se estaban haciendo públicas sobre las formas que éste había cobrado (aunque los elementos más perversos e inhumanos de su variante alemana todavía no eran conocidos por estas latitudes), caracterizar al movimiento argentino como una mala copia de aquel italiano, no tiene como sentido sino mostrarlo como más bastardo aún. Dice Halperin, al respecto: «... el peronismo no fue, sin duda, una forma de fascismo; fue por lo menos el resultado -o más bien el residuo, inesperado para todos y también para su creador y beneficiario- de una tentativa de reforma fascista de la vida política argentina ...»

Es a partir de esa sutileza, que vuelve Halperín a retomar la línea argumental que en ese momento era oficial y consagrada. Si el historiador abandonaría luego claramente los trazos gruesos de este criterio, a medida de que el peronismo ya fuera del poder se iría metamorfoseando impidiendo las analogías acríticas, la influencia política de la identificación peronismo-fascismo desde importantes sectores del poder político y las Fuerzas Armadas no cejaría y resultaría determinante en el largo proceso de inestabilidad política que mantendría a la primera pluralidad política proscripta de la legalidad política. Es decir: desandar la homologación entre el fenómeno argentino y el italiano llevaría al país veintiocho años de inestabilidad, de los cuales dieciocho con el peronismo proscripto y perseguido, y diez de caos, violencia y represión.

Sin embargo, en este apartado nos interesa revisar este argumento de 1956 donde Halperin Donghi trazaba una genealogía del fascismo en la Argentina que hace remontar a la década de 1930; entonces aparecían minorías que abogaban por la implementación del fascismo en el país, pero su importancia e influencia eran reducidas. Sólo en 1943, ya ante algún riesgo de apertura democrática (mientras en Europa se encontraba en su apogeo y se aprestaba para enfrentarse al resto del mundo) tomaría la iniciativa y se aprestaría a edificar la Argentina fascista. En esta experiencia prevalecería la interpretación conservadora del fascismo, y esto significaría una vuelta a supuestos valores tradicionales, como habían sido la España de Primo de Rivera o la Francia de Vichy. Lo que no encuentra nuestro autor es una correspondencia entre la supuesta tradición (basada en una primera instancia en lo religioso) que se restauraría y el campo sobre el cual restaurarla.

Podía ser considerada esta restauración como una amenaza para diversos sectores sociales, en particular la clase media superior y profesional, y desde esos sectores surgiría el primero de los grandes movimientos políticos de 1945: la resistencia argentina. Este concepto pretendía realizar la misma operación de establecer una analogía entre el proceso argentino de 1943-1955 con la situación europea de la Guerra Mundial. Aún salvando las diferencias abismales que hay entre los totalitarismos de la derecha europea y el caso local, y por lo tanto asumiendo que ha habido algunos elementos que permiten a algunos observadores decir que el primer peronismo fue todo lo fascista que se podía ser en el escenario de la posguerra, hablar de una Resistencia Argentina (así, con mayúsculas) entra deliberadamente en el terreno de la fantasía; más bien había surgido como operación propagandística por parte del antiperonismo más acérrimo, esta vez en el poder.

Es cierto que la relación entre el gobierno y la oposición tomó durante el peronismo, sobre todo desde 1951, características represivas y violentas y hubo episodios que, 
con cierto esfuerzo hermenéutico, pueden ser tomados como una forma de «resistencia», pero no pasaron de eventos, aunque resonantes, aislados y son tomados por la historiografía como actos de terrorismo. Sí la oposición política estaba diseminada por todo el territorio, y también es cierto que la actividad de propaganda debía ser clandestina, y fue constante y creciente y estaba distribuida de manera capilar. También es cierto que eran varios los partidos políticos que aún conservaban «fuerzas de choque», es decir, células destinadas a la defensa y la acción violenta, aunque no se verifican formaciones medianamente permanentes dedicadas al hostigamiento del régimen.

Lo concreto es que hacia julio de 1956, Halperín Donghi se acoplaba con toda su solvencia al discurso oficial y dominante. La identificación directa de peronismo y fascismo fue una opción que rápidamente el historiador corrigió, al evaluar de manera más compleja ese fenómeno político que fue el que surgió en la Argentina de 1943. Tal vez, la frase más famosa que nos deja, en ese sentido la escribiría años después, y es la que sirve como epígrafe a nuestro trabajo: el peronismo fue una revolución social: bastaba subirse a tranvía para darse cuenta de ello.

\section{Contorno y después ...}

La revista es considerada hoy por hoy como la piedra de toque de un cambio sustancial en las formas de aproximación a la literatura, porque, a partir de su irrupción el mundo de la crítica incorporará no sólo un nuevo lenguaje, nuevas lecturas y novedosas teorías - provenientes del amplio arco multidisciplinar- sino que interpretará el hecho literario como un hecho social, que es dado en un contexto determinado, y que resulta un campo propicio para examinar, como decía Osiris (Viñas), los problemas sociales, culturales, políticos. Los jóvenes denuncialistas de entonces desarrollaron, de una u otra manera, una premisa fundamental enunciada en 1964: la literatura y la realidad política (o la literatura y la política, directamente) van de la mano en la mirada que todos elaboren con respecto a la historia. Por ello, observar, reflexionar, desarmar el peronismo fue una primera manifestación de este hacer que perdurará a lo largo de los siglos XX y XXI y que hemos visto aquí, sucintamente, a través de las voces de Oscar Masotta y Halperin Donghi.

\section{BIBLIOGRAFÍA}

Altamirano, C. (2006): Intelectuales. Notas de Investigación. Buenos Aires: Grupo Editorial Norma. Altamirano, C., Sarlo, B. (1997): Ensayos argentinos. De Sarmiento a la vanguardia. Buenos Aires: Ariel.

Correas, C. (1991): La operación Masotta. Buenos Aires: Editorial Catálogos.

Croce, M. (1996): Contorno. Izquierda y proyecto cultural. Buenos Aires: Ediciones Colihue.

Echeverría, E. (1991): El matadero. En: Esteban Echeverría, Obras escogidas. Caracas: Biblioteca Ayacucho. 
Halperín Donghi, T. (1992): La larga agonía de la Argentina Peronista. Buenos Aires: Ariel.

Halperin Donghi, T. (1994): La Argentina en el callejón. Buenos Aires: Ariel.

Sarlo Sabajanes, B. (1983): «Los dos ojos de 'Contorno’». En: Revista Iberoamericana, 49, 125, 797-807.

Sigal, S., Eliseo V. (1982): «Perón, discurso político e ideología». En: Alain Rouquie (comp.): Argentina, hoy. México: Siglo XXI.

Spinelli, M. E. (2005): Los vencedores vencidos. El antiperonismo y la «revolución libertadora». Buenos Aires: Biblos.

Terán, O. (1986): «Rasgos de la cultura argentina en la década de 1950». En: En busca de la ideología argentina. Buenos Aires: Editorial Catálogos, 195-253.

Viñas, D. e I. (eds.) (2007): Contorno (1953-1959). Edición facsimilar. Buenos Aires: Biblioteca Nacional.

Viñas, D. (1964): Literatura argentina y realidad politica. Buenos Aires: Jorge Álvarez.

Viñas, D. (1999): Intelectuales, política y poder. Buenos Aires: Eudeba.

\section{RAZUMEVANJE PERONIZMA IN KULTURE V REVIJI CONTORNO (1953-1959)}

Ključne besede: revija Contorno, kultura, peronizem, Masotta, Halperin Donghi

Članek predstavi kritike, pisatelje in intelektualce, zbrane v uredništvu argentinske kulturne revije Contorno (1953-1959) - predstavnike generacije, ki je nastopila po t.i. »martinfierristih« (avtorjih, ki so objavljali v avantgardni reviji Martín Fierro) - in analizira njihov raznovrsten pogled na še danes ne povsem definiran družbeni in kulturni fenomen peronizma. Avtor posebej izpostavi intelektualno in politično videnje, ki ga je mogoče razbrati iz uredniškega zapisa v številki 7/8, in dva članka na to temo, ki prinašata pomembni perspektivi v okvirih literarne kritike na eni strani (Oscar Masotta) in zgodovine na drugi (Tulio Halperin Donghi). 